\title{
Watchdog censures food manufacturers on adverts targeted at children
}

Two health campaigning bodies have had their complaints upheld against food manufacturers who ran advertisements targeted at children.

Complaints were submitted by the Obesity Health Alliance (OHA) and the Children's Food Campaign to the Advertising Standards Authority (ASA) over three confectionery brands for online adverts for unhealthy food clearly aimed at children.

These were:

- Cadbury - a website for a joint promotion between Cadbury and the National Trust for Scotland which also provided downloadable content for Cadbury. The content included a storybook titled 'The Tale of the Great Easter Bunny', featuring children on an Easter egg hunt searching for purple Easter eggs, as well as an activity pack titled 'Eggciting activities' which featured Cadbury-branded purple eggs

- Chewits - four posts on the Chewits' Facebook page, hosted by cartoon character Chewie the Chewitsaurus. The theme of one post was celebration of GCSE results with Chewie; one focused on going 'back to school'; another focused on celebrating Roald Dahl Day with Chewits treats; and the final post was about celebrating International School Libraries Month

- Squashies - the 'advergame' app 'Squashies World' featured a game in which players were challenged to match pairs of Squashies by

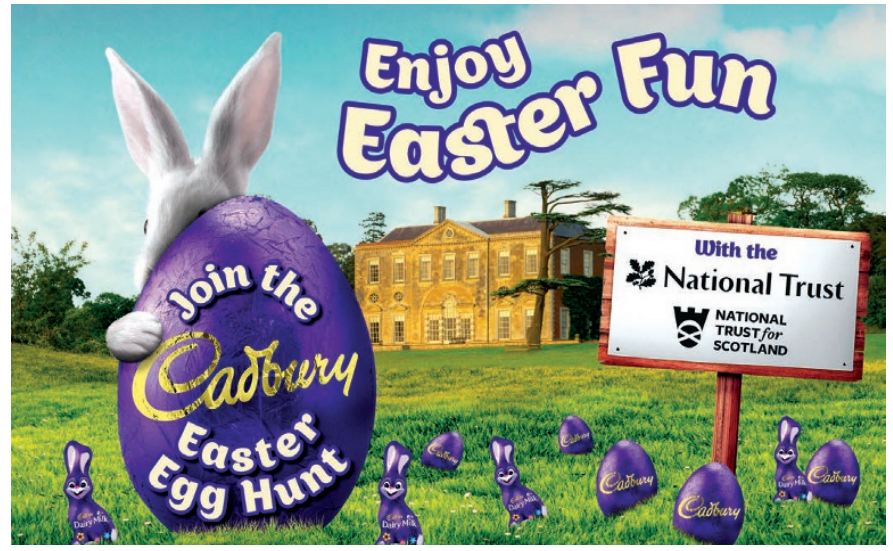

flicking them towards each other. The app's homepage featured images of three Squashies products and users who clicked on 'TEASE ME' were shown a page with text about Squashies and images of different versions of the sweet. Selecting 'PLAYTIME' opened an animation featuring cartoon images of anthropomorphised Squashies.

The OHA and the Children's Food Campaign said these campaigns had broken rules that were introduced by the Committee for Advertising Practice a year ago designed to protect children from junk food marketing.

The new rules for non-broadcast advertising, introduced in July 2017, place restrictions on the advertising of food and drink high in salt, sugar and fat (HFSS) to children online and via other non-broadcast media.

The OHA and the Children's Food Campaign said they did not believe the rules went far enough, particularly as they only applied when $25 \%$ of the audience was deemed to be children.

Caroline Cerny, OHA Lead, said: 'Whilst today's rulings should be celebrated, the complaints demonstrate the blatant ways in which the food and drink industry attempts to exploit loopholes in the rules.

'The Cadbury ruling, for example, revealed that the company doesn't actually hold any data to show that visitors to their website are predominately over 18 , but they state that 'it was unlikely that over $25 \%$ of its visitors were under the age of 16 . Worryingly, the Committee on Advertising Practice agreed with their assessment, despite no evidence to back this up.

'We knew that these rules would be difficult to monitor, and the fact that children continue to be exposed to various junk food marketing techniques proves us right.'

Barbara Crowther, Children's Food Campaign co-ordinator, added: 'Companies are not just breaching the rules, but clearly also ignoring the spirit of what those rules are there for, by deliberately targeting kids with apps, games and storybooks.

'We're pleased to see the ASA undertaking due diligence and upholding these complaints. However, it took six months for decisions to be reached, by which time the advertising campaigns had long ago concluded. The companies in question weren't penalised in any way, and children remained largely unprotected from the harmful effects of junk food marketing.' 